\title{
Hochenergetische Konversionselektronen und das Niveauschema von ${ }^{200} \mathrm{Hg}$ *
}

\author{
W. Mampe, T. von Egidy, W. Kaiser \\ und K. SCHRECKENBACH \\ Physik-Department der Technischen Universität München \\ (Z. Naturforsch. 26 a, 405-418 [1971] ; eingegangen am 15. Dezember 1970) \\ Herrn Prof. Dr. H. Maier-Leibnitz zum 60. Geburtstag gewidmet
}

\begin{abstract}
Approximately 165 conversion electron lines from slow neutron capture in ${ }^{199} \mathrm{Hg}$ have been measured with the beta spectrometer at the reactor FRM between $900 \mathrm{keV}$ and $8 \mathrm{MeV}$. The high energy $\mathrm{K}$-conversion coefficients of $\mathrm{El}$ and $\mathrm{Ml}$ transitions are separated distinctly and agree very well with theoretical calculations, which have been performed by several groups recently. High energy L-conversion electron coefficients have been measured for the first time with such a degree of accuracy that different multipole orders are separated and a comparison with theoretical values is possible. 6 known levels above $2 \mathrm{MeV}$ have been assigned $1^{+}$and 14 new levels have been established. 145 lines have been placed into the level scheme and new level energies have been calculated. A new $0^{+}$level has been found at $1856.97 \pm 0.1 \mathrm{keV}$.
\end{abstract}

\section{Einleitung}

Die ersten Messungen von Konversionselektronen mit Energien bis zu $9 \mathrm{MeV}^{1-7}$ und die damit mög. liche experimentelle Bestimmung von Konversionskoeffizienten in diesem Energiebereich führten sehr bald zu theoretischen Berechnungen von hochenergetischen Konversionskoeffizienten durch $\mathrm{CHURCH}^{8,9}$, Dragoun ${ }^{10}$, Trusov ${ }^{11}$ und Carroll und O'ConNELL ${ }^{12,13}$. Dabei ergab sich innerhalb der großen Fehler der experimentellen Koeffizienten für mittelschwere Kerne eine zufriedenstellende Übereinstimmung mit der Theorie. Zuvor waren theoretische Konversionskoeffizienten nur bis $2,5 \mathrm{MeV}$ berechnet worden ${ }^{14}$. Eine eindeutige Unterscheidung der Multipolordnungen über $6 \mathrm{MeV}$ war jedoch schwierig ${ }^{15,16}$. Die hochenergetischen Konversionskoeffizienten schwerer Kerne sollten die Prüfung der theo-

Sonderdruckanforderungen an W. MAMPE, Physik-Department E 18, Technische Universität München, D-8000 München 2, Arcisstraße 21.

* Auszug aus der Dissertation an der Fakultät für Allgemeine Wissenschaften der Technischen Universität München von Walter Mampe (1971)

1 Th. W. Elze, T. v. Egidy u. E. Bieber, Z. Physik 184, 229 [1965].

2 Th. W. Elze, Z. Physik 194, 280 [1966].

3 T. v. Egidy, E. Bieber u. Th. W. Elze, Z. Physik 195, 489 [1966].

4 T. v. Egidy u. W. Kaiser, Z. Physik 201, 378 [1967].

5 B. C. Dutta, T. v. Egidy, Th. W. Elze u. W. Kaiser, Z. Physik 207, 153 [1967].

6 Yu. N. Panin, V. I. Pelekhov u. T. I. Turguntaev, Izv. Akad. Nauk SSSR, Ser. Fiz. 33, 670 [1969]. retischen Ansätze zu ihrer Berechnung besser ermöglichen, da sowohl die Größe der Konversionskoeffizienten als auch ihre Abhängigkeit von der Multipolordnung mit $Z$ zunimmt. Ebenso wächst die Empfindlichkeit der Koeffizienten auf Durchdringungseffekte (Penetration) mit der Ordnungszahl und der Energie ${ }^{9}$. In diesem Gebiet scheint die Messung der Durchdringungseffekte und damit der Kernparameter $\lambda$ mit Hilfe sehr genau bestimmter Konversionskoeffizienten möglich zu sein. Die Kernparameter $\lambda$ geben Auskunft über die Eigenschaften hochangeregter Kernzustände. Nach $\mathrm{CHURCH}^{9}$ ist der Betrag der Kernstruktureffekte zum Konversionskoeffizienten in der Regel kleiner als $10 \%$, er kann aber auch bei erlaubten $\gamma$-Übergängen möglicherweise erheblich über $10 \%$ liegen.

${ }^{200} \mathrm{Hg}$ mit $Z=80$ ist für die Untersuchungen besonders geeignet, da es sehr intensive Übergänge bei

7 Yu. N. Panin u. V. I. Pelekhov, Izv. Akad. Nauk SSSR, Ser. Fiz. 34, 804 [1970].

8 E. Church u. J. Weneser, Bull. Amer. Phys. Soc. 13, 893 [1968].

9 E. Church, Phys. Lett. 28 B, 168 [1968].

10 O. Dragoun, Nucl. Phys. A 132, 374 [1969].

11 V. F. Trusov, Sov. J. Nucl. Phys. 9, 429 [1969].

12 C. O. Carroll u. R. F. O’Connell, Nucl. Phys. 80, 500 [1966].

13 C. O. Carroll u. R. F. O'Connell, Nucl. Phys. A 125, 637 [1969].

14 L. Sliv u. I. Band, in: K. Siegbahn, Alpha-, Beta- and Gamma-ray spectroscopy, North Holland Publ. Comp., Amsterdam 1965.

15 R. K. Smither, Phys. Lett. 25 B, 128 [1967].

16 J. A. Moragues, W. Gelletly u. M. A. J. Mariscotti, Phys. Lett. 27 B, 441 [1968]. 
hohen Energien hat und ${ }^{199} \mathrm{Hg}$ einen großen Neutroneneinfangquerschnitt und hohe Neutronenbindungsenergie hat.

Die Struktur von ${ }^{200} \mathrm{Hg}$, das 2 Protonen und 6 Neutronen weniger besitzt als der doppelt magische Kern ${ }^{208} \mathrm{~Pb}$, ist theoretischen Berechnungen nur schwer zugänglich. ${ }^{200} \mathrm{Hg}$ liegt im Übergangsbereich zwischen sphärischen und deformierten Kernen. Bei höheren Anregungsenergien werden deformierte $\mathrm{Zu}$ stände erwartet ${ }^{43}, 44$. Deshalb sind möglichst vollständige experimentelle Ergebnisse über das Niveauschema die Voraussetzung, um die verschiedenen theoretischen Ansätze überprüfen zu können. Die Übergänge des gg-Kerns ${ }^{200} \mathrm{Hg}$ und sein Niveauschema wurden von mehreren Autoren studiert: nach dem Zerfall von ${ }^{200} \mathrm{Au}{ }^{17-21}$, dem Zerfall von ${ }^{200} \mathrm{Tl}{ }^{22-25}$ und den Reaktionen ${ }^{199} \mathrm{Hg}(\mathrm{n}, \gamma)^{200} \mathrm{Hg}$ (s. ${ }^{26-32}$ ) sowie ${ }^{199} \mathrm{Hg}(\mathrm{n}, \mathrm{e}){ }^{200} \mathrm{Hg}{ }^{26,33}$. Die meisten Arbeiten beschäftigen sich mit den tiefer liegenden Zuständen bis 1,5 MeV und führen in diesem Gebiet zu einigermaßen befriedigender Übereinstimmung mit den Vorhersagen des theoretischen Modells ${ }^{34-36}$. In Ergänzung hierzu und im Anschluß an unsere frühere Arbeit ${ }^{33}$ über Konversionselektronen bis $2,2 \mathrm{MeV}$ sollten die Konversionsübergänge bis zur Neutronenbindungsenergie hin untersucht werden, um Spin und Paritätszuordnungen für die höherliegenden Niveaus festlegen zu können.

\section{Experimentelle Methode und Ergebnisse}

Die Messungen wurden mit dem Beta-Spektrometer ${ }^{2,3,37}$ am Forschungsreaktor FRM bei München durchgeführt. Bei der Reaktorleistung von $4 \mathrm{MW}$ be-

17 J. C. Roy u. R. A. Roy, Can. J. Phys. 37, 385 [1959].

18 R. K. Girgis, R. A. Ricci u. R. van Lieshout, Nucl. Phys. 11, 889 [1960].

19 O. W. B. Schult, W. R. Kane u. E. Der Mateosian, Phys. Rev. 170, 1055 [1968].

20 K. Sakai u. P. J. Daly, Nucl. Phys. A 118, 361 [1968].

21 H. Ton, G. H. Dulfer, J. Brasz, R. Kroondijk u. J. Blok, Nucl. Phys. A 153, 129 [1970].

22 M. Sakai, H. Ikegami, T. Yamazaki u. K. Saito, Nucl. Phys. 65, 177 [1965].

23 R. Béraud, I. Berkes, J. Danière, R. Haroutunian, M. Lévy, G. Marest u. R. Rougny, Phys. Rev. 188, 1958 [1969].

24 M. Sakai, J. Phys. Soc. Japan 26, 879 [1969].

25 M. Sakai, H. Kawakami u. K. Saito, J. Phys. Soc. Japan 28, 542 [1970].

26 L. V. Groshev, A. M. Demidov, V. A. Ivanov, N. V. LutSenko u. V. I. Pelekhov, Izv. Akad. Nauk SSSR, Ser. Fiz. 27, 1377 [1963].

27 B. P. Maier, U. Gruber, H. R. Косh u. O. W. B. Schult, Z. Physik 185, 478 [1965]. trägt der Fluß am Ort des Targets ca. $6,4 \cdot 10^{12} \mathrm{n} / \mathrm{cm}^{2}$ -sec. Das Target bestand aus angereichertem $\mathrm{HgS} * *$, da das übliche $\mathrm{HgO}$ bei der Bestrahlung im Reaktor sublimiert. Wegen dieser ${ }^{199} \mathrm{Hg}$-Anreicherung erwartet man keine Konversionslinien anderer Isotope. $13 \mathrm{mg}$ $/ \mathrm{cm}^{2} \mathrm{HgS}$ waren auf $10 \times 8 \mathrm{~mm}^{2}$ einer $1 \mathrm{mg} / \mathrm{cm}^{2}$ starken Al-Folie sedimentiert. Die HgS-Schicht war mit einer $0,2 \mathrm{mg} / \mathrm{cm}^{2}$ starken $\mathrm{Al}$-Folie abgedeckt und mit $3 \mathrm{t} / \mathrm{cm}^{2}$ gepreßt worden. Eine Beimischung von $1 \mathrm{mg}$ UHU-Alleskleber in Benzol gelöst verlieh der $\mathrm{HgS}$ Schicht zusätzliche Stabilität.

Das Spektrum wurde zwischen 480 und $2300 \mathrm{keV}$ zweimal mit 1 Minute pro Meßpunkt durchgefahren, zwischen $2200 \mathrm{keV}$ und $8000 \mathrm{keV}$ viermal und abschnittsweise fünfmal mit 5 und teilweise 10 Minuten pro Meßpunkt. Die Impulsauflösung war etwa $0,3 \%$. Abbildung 1 zeigt einen Teil des Spektrums. Die Auswertung erfolgte mit einem Fitprogramm, das den Elektronenlinien Gauß-Kurven mit exponentiell abfallendem Teil bei niederen Energien anpaßt ${ }^{38}$. Die Qualität des Fits zeigt sich bei den hochenergetischen $M$-Linien, die an der Flanke der $L$-Linien gefunden werden. Der Energiebereich zwischen $480 \mathrm{keV}$ und $2300 \mathrm{keV}$ war früher mit besserer Auflösung bei dünnerem Target gemessen worden ${ }^{33}$ und sollte hier nur den Umrechnungsfaktor für die relative Intensitätseichung liefern.

Alle Ergebnisse sind in Tab. 1 aufgeführt. Die angegebenen Energiefehler enthalten Fitfehler, Eichfehler und den Fehler von $2,5 \cdot 10^{-4}$, der durch die Unlinearität des Potentiometers zur Magnetstromeinstellung bedingt ist. Die relative Konversionselektronenintensität $I_{\mathrm{e}}$ in Spalte 7 entspricht innerhalb $\pm 20 \%$ der absoluten Intensität von $I_{\mathrm{e}}$ pro $10^{4}$ eingefangener Neutronen. Der angegebene Fehler der Intensität (Spalte 8) ist im wesentlichen statistischer Art. Die $\gamma$-Intensitäten in Spalte 9 von Übergängen bis $3300 \mathrm{keV}$ wurden aus der Arbeit von

28 O. W. B. Schult, W. R. Kane, M. A. J. Mariscotti u. J. M. Simić, Phys. Rev. 164, 1548 [1967].

29 G. A. Barthomolew, S. I. H. Nagvi, M. R. Gunye u. E. D. EARle, Can. J. Phys. 45, 1517 [1967].

30 G. A. Bartholomew, M. R. Gunye u. E. D. Earle, Can. J. Phys. 45, 2063 [1967].

31 E. R. Rae, W. R. Moyer, R. R. Fullwood u. J. L. AnDREWS, Phys. Rev. 155, 1301 [1967].

32 N. C. Rasmussen, V. J. Orphan, Y. Hukai u. T. Inouye, priv. Mitteilung in: Nuclear Data A 5, 384 [1969].

33 O. W. B. Schult, W. Kaiser, W. Mampe u. T. v. Egidy, Z. Physik 218, 95 [1969].

34 N. Bijedić, Phys. Lett. 16, 47 [1965].

35 G. Alaga u. G. Ialongo, Phys. Lett. 22, 619 [1966].

36 A. Covello u. G. Sartoris, Nucl. Phys. A 104, 189 [1967].

37 T. v. Egidy, Ann. Physik 9, 221 [1962].

* Vom Hersteller (Oak Ridge) angegebene Isotopenzusam. mensetzung: ${ }^{196} \mathrm{Hg}: 0,05 \%,{ }^{198} \mathrm{Hg}: 2,3 \%,{ }^{199} \mathrm{Hg}: 83,45 \%$, ${ }^{200} \mathrm{Hg}: 6,1 \%,{ }^{201} \mathrm{Hg}: 2,36 \%,{ }^{202} \mathrm{Hg}: 4,76 \%,{ }^{204} \mathrm{Hg}: 9,03 \%$.

${ }^{38}$ T. v. Egidy u. Th. W. Elze, FRM-Bericht, T.U. München 1966. 


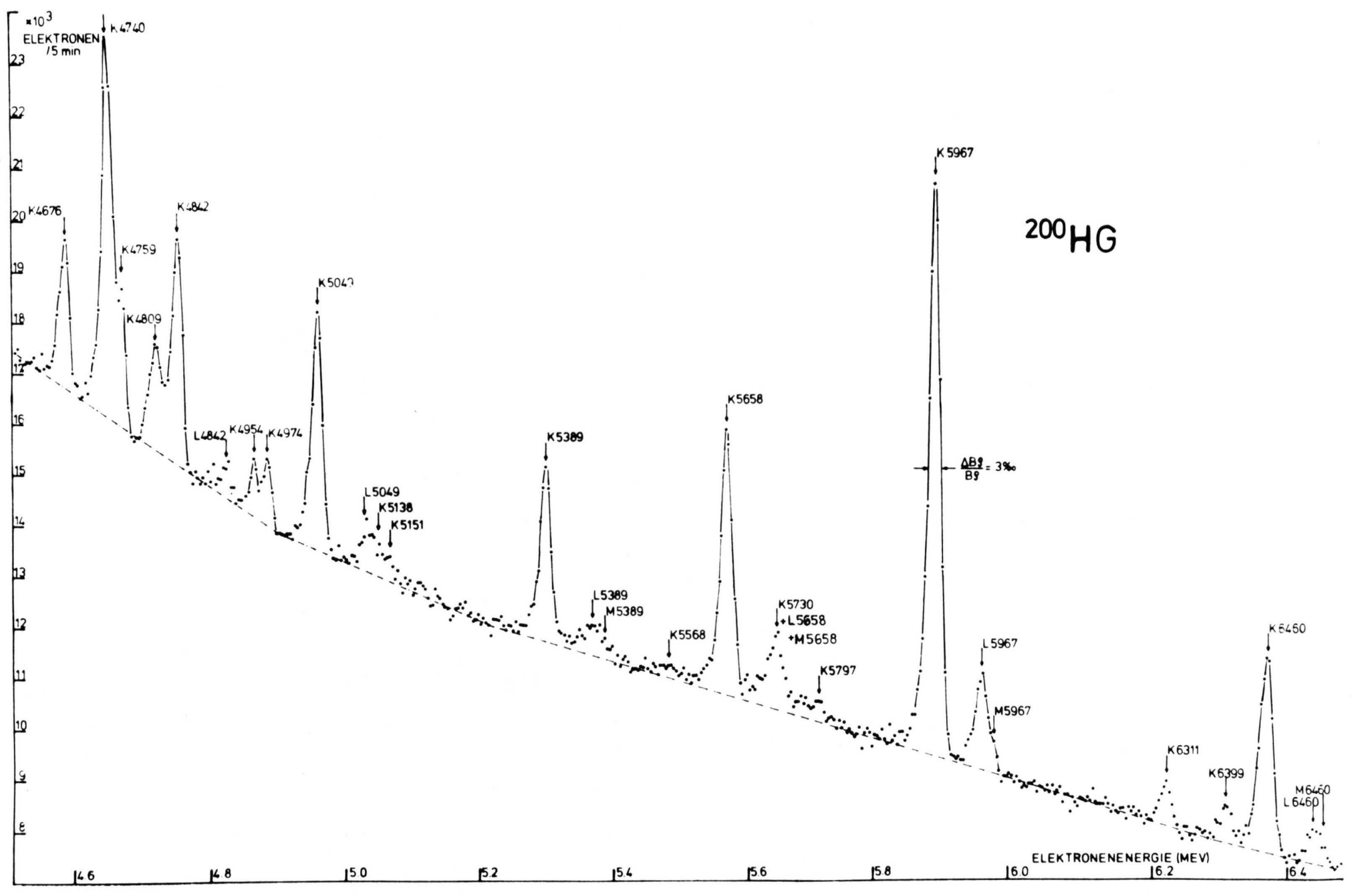

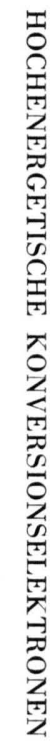

Abb. 1. Teil des hochenergetischen Konversionselektronenspektrums von ${ }^{200} \mathrm{Hg}$. 
Tab. 1. Konversionselektronen von ${ }^{200} \mathrm{Hg}$. Die Gamma-Energien und -Intensitäten sind Ref. ${ }^{28}$ entnommen, wenn nicht durch einen Index eine andere Referenz angezeigt wird ( $m=27, g=26, r=32, a=31$ ). Mit „e“ indizierte Gamma-Linien wurden zur Energieeichung benützt. Der Index „b“ bezeichnet im Niveauschema eingeordnete Linien. Durch "p“ gekennzeichnete Linien sind als wahrscheinliche Übergänge in das Niveauschema aufgenommen. Die relativen Konversionselektronenintensitäten $I_{\mathrm{e}}$ sind auf die relativen Gamma-Intensitäten $I$ bezogen, wobei die K-Konversionslinie des 579 keV-Übergangs mit der bekannten Multipolarität E2 zugrunde gelegt ist. Die Abkürzungen in Spalte 17 besagen: $w=$ schwach, $c=$ Liniengruppe, $q=$ fragliche Linie, $-L:$ die Intensität der überlagerten $L$-Linie ist abgezogen, $+L$ : die In tensität der überlagerten L-Linie ist nicht abgezogen.

\begin{tabular}{|c|c|c|c|c|c|c|c|c|c|c|c|c|c|c|c|c|}
\hline$E$ & $\Delta E_{\gamma}$ & Schale & $E_{\mathrm{e}}$ & $\Delta E_{\mathrm{e}}$ & $E_{\text {trans }}$ & $\begin{aligned} & I_{\mathrm{e}} \\
\times & 100\end{aligned}$ & $\frac{\Delta I_{\mathrm{e}}}{I_{\mathrm{e}}}$ & $I_{\gamma}$ & $\frac{\Delta I_{\gamma}}{I_{\gamma}}$ & $\begin{array}{r}\alpha_{\exp } \\
\times 100\end{array}$ & $\begin{array}{c}\Delta \alpha_{\exp } \\
\alpha_{\exp }\end{array}$ & \multicolumn{3}{|c|}{$\begin{array}{c}\text { Theoret. Konversions- } \\
\text { koeffizient } \times 100\end{array}$} & \multirow[t]{2}{*}{ Multipolarität } & \multirow[t]{2}{*}{ Kommentar } \\
\hline$[\mathrm{keV}]$ & {$[\mathrm{keV}]$} & & {$[\mathrm{keV}]$} & {$[\mathrm{keV}]$} & {$[\mathrm{keV}]$} & & {$[\%]$} & & {$[\%]$} & & {$[\%]$} & $E 1$ & E2 & M1 & & \\
\hline 1 & 2 & 3 & 4 & 5 & 6 & 7 & 8 & 9 & 10 & 11 & 12 & 13 & 14 & 15 & 16 & 17 \\
\hline $904,6^{\mathrm{m}}$ & 1,5 & $\mathrm{~K}$ & 817,5 & 2,1 & 900,6 & 0,16 & 30 & & & & & 0,23 & 0,60 & 1,66 & & b \\
\hline $935,2 \mathrm{~m}$ & 1,0 & $\mathrm{~K}$ & 854,3 & 0,6 & 937,4 & 0,15 & 70 & & & & & 0,22 & 0,56 & 1,54 & & \\
\hline 977,1 & 1,5 & $\mathrm{~K}$ & 992,0 & 0,7 & 975,1 & 0,41 & 15 & 0,42 & - & 1,0 & - & 0,20 & 0,51 & 1,34 & M1, E2 & \\
\hline \multirow[t]{3}{*}{$1011,5^{\mathrm{m}}$} & 1,5 & $\mathrm{~K}$ & 931,2 & 0,6 & 1014,3 & 0,19 & 10 & $<0,1$ & - & $>1,9$ & - & 0,20 & 0,50 & 1,28 & (M1) & \\
\hline & & $\mathrm{K}$ & 946,4 & - & 1029,4 & $<0,1$ & - & & & & & & & & & \\
\hline & & $\mathrm{K}$ & 959,6 & 1,7 & 1042,7 & 0.15 & 30 & & & & & & & & & $\mathrm{w}, \mathrm{c}, \mathrm{p}$ \\
\hline \multirow[t]{2}{*}{$1050^{\mathrm{m}}$} & 2,4 & $\mathrm{~K}$ & 972,0 & 0,7 & 1055,1 & 0,15 & 50 & 0,6 & - & 0,25 & - & 0,18 & 0,47 & 1,18 & (E2, E 1) & $\mathrm{w}$ \\
\hline & & $\mathrm{K}$ & 1040,9 & 1,3 & 1124,0 & 0,22 & 10 & & & & & & & & & $\mathrm{c}, \mathrm{b}$ \\
\hline \multirow[t]{3}{*}{1147,33} & 0,2 & $\mathrm{~K}$ & 1064,9 & 0,3 & 1148,0 & 0,68 & 5 & 1,7 & 10 & 0,40 & 15 & 0,152 & 0,38 & 0,89 & $\mathrm{E} 2$ & $\mathrm{p}$ \\
\hline & & $\mathrm{K}$ & 1080,8 & 0,4 & 1163,9 & 0,18 & 50 & $<0,3$ & - & $>0,6$ & - & 0,148 & 0,37 & 0,86 & $\mathrm{M} 1,(+\mathrm{E} 2)$ & $\mathrm{b}$ \\
\hline & & $\mathrm{K}$ & 1098,8 & 0,5 & 1181,9 & 0,41 & 60 & $<0,3$ & - & $>1,4$ & - & 0,144 & 0,36 & 0,83 & (M1) & \\
\hline 1202,55 & 0,2 & $\mathrm{~K}$ & 1119,8 & 0,4 & 1202,9 & 2,0 & 10 & 3,5 & 20 & 0,57 & 25 & 0,140 & 0,35 & 0,79 & $\mathrm{M} 1+(50 \pm 30) \% \mathrm{E} 2$ & b \\
\hline 1206,0 & 0,2 & $\mathrm{~K}$ & 1122,5 & 0,4 & 1205,6 & 2,73 & 10 & 3,5 & 20 & 0,78 & 25 & 0,139 & 0,34 & 0,78 & M1 & b \\
\hline \multirow[t]{3}{*}{$1225,73^{\mathrm{e}}$} & 0,1 & $\mathrm{~K}$ & 1142,5 & 0,3 & 1225,6 & 2,36 & 5 & 2,9 & 10 & 0,81 & 12 & 0,136 & 0,33 & 0,76 & M1 & b \\
\hline & & $\mathrm{L}$ & 1209,8 & 0,6 & & 0,32 & 15 & & & 0,11 & 20 & 0,021 & 0,06 & 0,13 & & \\
\hline & & $\mathrm{K}$ & 1157,1 & 0,7 & 1240,2 & 0,20 & 80 & $<0,3$ & - & $>0,7$ & - & 0,133 & 0,33 & 0,73 & (M1) & \\
\hline 1254,18 & 0,2 & K & 1168,7 & 1,5 & 1251,8 & 0,58 & 12 & 1,7 & 15 & 0,34 & 20 & 0,131 & 0,32 & 0,71 & E 2 & b \\
\hline \multirow[t]{2}{*}{1263,15} & 0,10 & $\mathrm{~K}$ & 1179,8 & 0,3 & 1262,9 & 3,05 & 3 & 4,3 & 10 & 0,71 & 12 & 0,129 & 0,32 & 0,70 & M1 & b \\
\hline & & $\mathrm{L}$ & 1248,0 & 0,5 & & 0,43 & 10 & & & 0,10 & 12 & 0,020 & 0,05 & 0,12 & & \\
\hline \multirow[t]{3}{*}{1273,7} & 0,15 & K & 1190,3 & 0,3 & 1273,4 & 1,48 & 5 & 2,5 & 10 & 0,60 & 12 & 0,127 & 0,31 & 0,69 & $\mathrm{M} 1+\left(25 \pm_{10}^{65}\right) \% \mathrm{E} 2$ & $+L 1205$ \\
\hline & & $\mathrm{L}$ & 1258,6 & 0,4 & & 0,23 & 10 & & & 0,09 & 15 & 0,020 & 0,05 & 0,12 & & $-M 1273$ \\
\hline & & $\mathrm{K}$ & 1240,1 & 0,9 & 1323,2 & 0,14 & 30 & $<0,3$ & - & $>0,5$ & - & 0,120 & 0,29 & 0,62 & M 1 & $\mathrm{~b}$ \\
\hline 1349,46 & 0,3 & $\mathrm{~K}$ & 1266,2 & 0,4 & 1349,3 & 0,52 & 8 & 1,3 & 20 & 0,40 & 25 & 0,115 & 0,28 & 0,59 & $\mathrm{E} 2+(40 \pm 30) \% \mathrm{M} 1$ & \\
\hline 1363,06 & 0,25 & $\mathrm{~K}$ & 1279,9 & 0,3 & 1363,0 & 0,83 & 10 & 1,5 & 20 & 0,55 & 20 & 0,113 & 0,28 & 0,58 & $\mathrm{M} 1+<70 \% \mathrm{E} 2$ & b \\
\hline 1384,9 & & $\mathrm{~K}$ & 1302,1 & 0,6 & 1385,3 & 0,17 & 10 & 0,35 & 40 & 0,49 & 45 & 0,110 & 0,27 & 0,55 & $\mathrm{M} 1, \mathrm{E} 2$ & b \\
\hline \multirow[t]{5}{*}{1407,80} & 0,15 & $\mathrm{~K}$ & 1325,3 & 0,4 & 1408,4 & 0,94 & 5 & 2,2 & 15 & 0,43 & 15 & 0,107 & 0,26 & 0,53 & $\mathrm{M} 1+(40 \pm 40) \% \mathrm{E} 2$ & b \\
\hline & & $\mathrm{L}$ & 1393,5 & 1,0 & & 0,11 & 20 & & & 0,05 & 30 & 0,015 & 0,04 & 0,08 & & \\
\hline & & $\mathrm{K}$ & 1337,4 & 1,2 & 1420,5 & 0,11 & 20 & $<0,5$ & - & $>0,22$ & - & 0,105 & 0,25 & 0,52 & $\mathrm{E} 2, \mathrm{M} 1$ & b \\
\hline & & $\mathrm{K}$ & 1349,2 & 0,5 & 1432,3 & 0,12 & 25 & $<0,5$ & - & $>0,24$ & - & 0,104 & 0,25 & 0,51 & $\mathrm{E} 2, \mathrm{M} 1$ & $\mathrm{~b},-L 1363$ \\
\hline & & $\mathrm{K}$ & 1383,9 & 0,6 & 1467,0 & 0,25 & 12 & $<0,8$ & - & $>0,32$ & - & 0,100 & 0,24 & 0,48 & $\mathrm{M} 1+<50 \% \mathrm{E} 2$ & b \\
\hline \multirow[t]{2}{*}{ (1491) } & & $\mathrm{K}$ & 1406,5 & 0,7 & 1489,6 & 0,25 & 20 & 1,0 & 30 & 0,25 & - & 0,098 & 0,23 & 0,46 & $\mathrm{E} 2$ & b \\
\hline & & $\mathrm{K}$ & 1419,7 & 1,1 & 1502,8 & 0,09 & 40 & $<0,6$ & - & $>0,15$ & - & 0,097 & 0,23 & 0,45 & $\mathrm{E} 2, \mathrm{M} 1$ & b \\
\hline \multirow[t]{2}{*}{1515} & & $\mathrm{~K}$ & 1431,8 & 0,4 & 1514,9 & 0,53 & 5 & 0,7 & 30 & 0,69 & 35 & 0,094 & 0,23 & 0,44 & M1 & $\mathrm{b},-L 1447$ \\
\hline & & $\mathrm{K}$ & 1463,0 & 0,5 & 1546,1 & 0,41 & 30 & $<0,7$ & - & $>0,58$ & - & 0,093 & 0,22 & 0,42 & M1 & \\
\hline 1558,0 & 0,25 & $\mathrm{~K}$ & 1474,5 & 1,4 & 1557,6 & 0,28 & 60 & 0,65 & 25 & 0,43 & 40 & 0,090 & 0,22 & 0,41 & $\mathrm{M} 1(+<80 \% \mathrm{E} 2)$ & $\mathrm{b},-\mathbf{G} 1489$ \\
\hline \multirow[t]{2}{*}{1570,33} & 0,2 & $\mathrm{~K}$ & 1487,6 & 0,3 & 1570,7 & 2,52 & 3 & 6,8 & 10 & 0,37 & 12 & 0,088 & 0,212 & 0,40 & M1 & b \\
\hline & & $\mathrm{L}$ & 1555,1 & 0,5 & & 0,40 & 10 & & & 0,067 & 15 & 0,014 & 0,036 & 0,069 & & \\
\hline 1604,65 & 0,3 & $\mathrm{~K}$ & 1523,4 & 0,5 & 1606,5 & 0,29 & 10 & 0,8 & 25 & 0,36 & 30 & 0,086 & 0,203 & 0,39 & $\mathrm{M} 1(+<80 \% \mathrm{E} 2)$ & \\
\hline
\end{tabular}




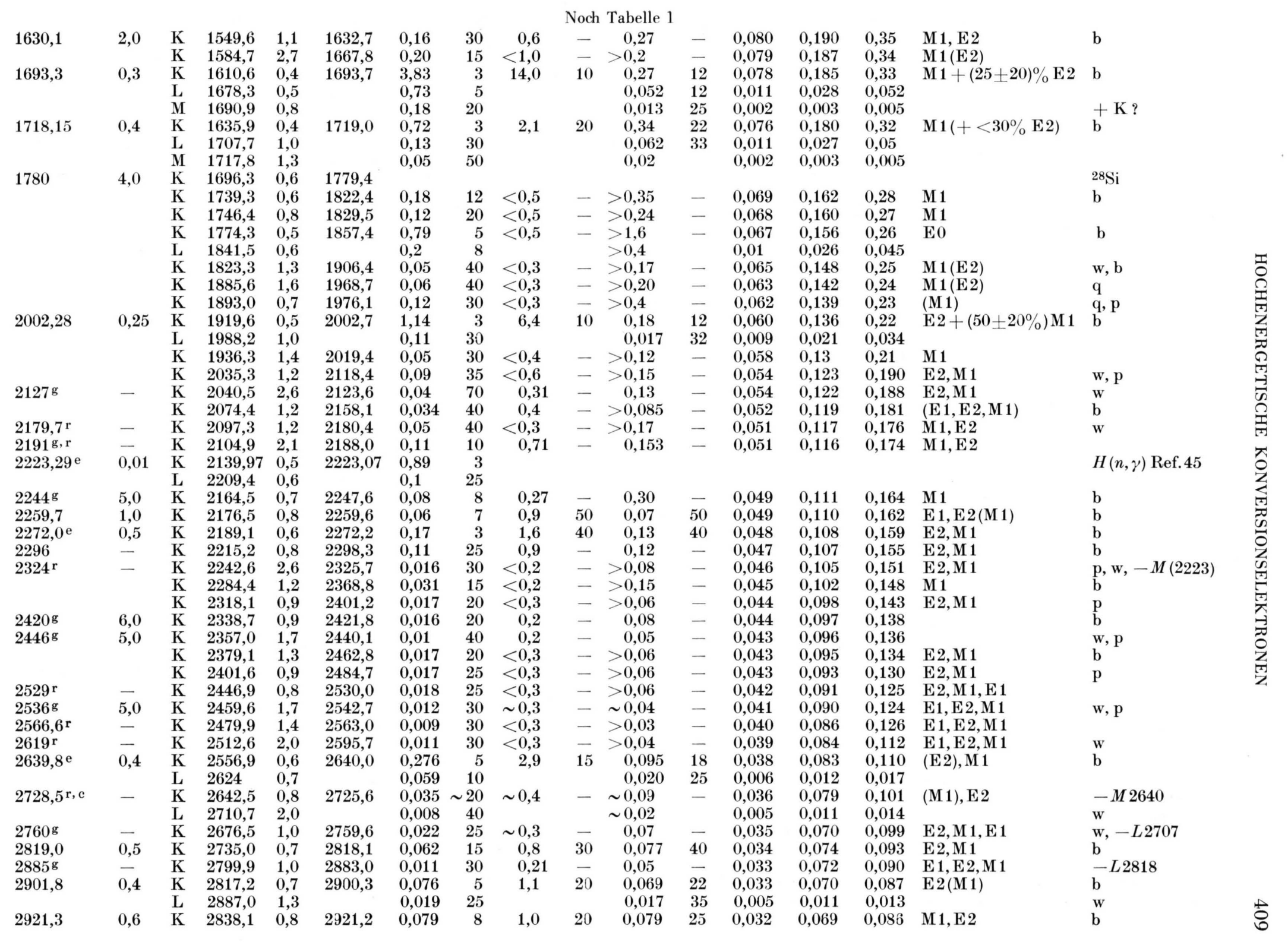


Noch Tabelle 1

\begin{tabular}{|c|c|c|c|c|c|c|c|c|c|c|c|c|c|c|c|c|}
\hline \multirow{3}{*}{$\begin{array}{c}E \\
{[\mathrm{keV}]} \\
1\end{array}$} & \multirow{2}{*}{$\begin{array}{c}\Delta E_{\gamma} \\
{[\mathrm{keV}]}\end{array}$} & \multirow{2}{*}{ Schale } & \multirow{2}{*}{$\begin{array}{c}E_{\mathrm{e}} \\
{[\mathrm{keV}]}\end{array}$} & \multirow{2}{*}{$\begin{array}{c}\Delta E_{\mathrm{e}} \\
{[\mathrm{keV}]}\end{array}$} & \multirow{2}{*}{$\begin{array}{l}E_{\text {trans }} \\
{[\mathrm{keV}]}\end{array}$} & \multirow{2}{*}{$\begin{array}{c}I_{\mathrm{e}} \\
\times 100\end{array}$} & \multirow{2}{*}{$\begin{array}{l}\frac{\Delta I_{\mathrm{e}}}{I_{\mathrm{e}}} \\
{[\%]}\end{array}$} & \multirow[t]{2}{*}{$I_{\gamma}$} & \multirow{2}{*}{$\begin{array}{l}\frac{\Delta I_{\gamma}}{I_{\gamma}} \\
{[\%]}\end{array}$} & \multirow{2}{*}{$\begin{array}{l}\alpha_{\exp } \\
\times 100\end{array}$} & \multirow{2}{*}{$\begin{array}{c}\frac{\Delta \alpha_{\exp }}{\alpha_{\exp }} \\
{[\%]}\end{array}$} & \multicolumn{3}{|c|}{ Theoret. Konversions- } & \multirow[t]{2}{*}{ Multipolarität } & \multirow[t]{2}{*}{ Kommenta } \\
\hline & & & & & & & & & & & & E 1 & $\mathrm{E} 2$ & M1 & & \\
\hline & 2 & 3 & 4 & 5 & 6 & 7 & 8 & 9 & 10 & 11 & 12 & 13 & 14 & 15 & 16 & 17 \\
\hline 2984,6 & - & $\mathrm{K}$ & 2901,3 & 1,0 & 2984,4 & 0,022 & 15 & 0,4 & 40 & 0,055 & 50 & 0,031 & 0,067 & 0,081 & $\mathrm{E} 2, \mathrm{E} 1, \mathrm{M} 1$ & $\mathrm{~b},-L 2921$ \\
\hline $2993 \mathrm{~g}$ & - & $\mathrm{K}$ & 2909,6 & 1,3 & 2992,7 & 0,020 & 30 & 0,2 & - & 0,10 & - & 0,031 & 0,066 & 0,079 & M1, E 2 & w \\
\hline 3050 & - & $\mathrm{K}$ & 2969,1 & 0,9 & 3052,2 & 0,029 & 20 & 0,6 & 20 & 0,049 & 35 & 0,030 & 0,064 & 0,077 & $\mathrm{E} 2, \mathrm{E} 1, \mathrm{M} 1$ & b \\
\hline 3073 & - & $\mathrm{K}$ & 2991,4 & 0,9 & 3074,5 & 0,028 & 10 & 0,5 & 25 & 0,056 & 30 & 0,030 & 0,063 & 0,076 & E2(M 1) & b \\
\hline \multirow[t]{2}{*}{$3104^{\mathrm{g}}$} & - & $\mathrm{K}$ & 3021,5 & 1,4 & 3104,6 & 0,011 & 30 & 0,38 & - & 0,029 & - & 0,030 & 0,062 & 0,073 & E1 & $\mathrm{w}$ \\
\hline & & $\mathrm{K}$ & 3039,4 & 1,5 & 3122,5 & 0,012 & 20 & $<0,15$ & - & $>0,08$ & - & 0,030 & 0,061 & 0,072 & M1 & $\mathrm{b}$ \\
\hline \multirow{2}{*}{$3185,9^{\mathrm{e}}$} & 0,5 & $\mathrm{~K}$ & 3102,7 & 0,9 & 3185,8 & 0,163 & 4 & 2,4 & 15 & 0,068 & 17 & 0,029 & 0,060 & 0,070 & M1 & b \\
\hline & & $\mathrm{L}$ & 3171,9 & 1,0 & & 0,023 & 15 & & & 0,010 & 25 & & & & & \\
\hline 3216,0 & 0,8 & $\mathrm{~K}$ & 3134,7 & 0,9 & 3217,8 & 0,041 & 8 & 0,7 & 25 & 0,057 & 30 & 0,028 & 0,059 & 0,068 & & $\mathrm{p}$ \\
\hline 3269,0 & 1,0 & $\mathrm{~K}$ & 3185,7 & 0,9 & 3268,8 & 0,049 & 7 & 0,7 & 20 & 0,070 & 25 & 0,028 & 0,057 & 0,066 & M1 & $\mathrm{b}$ \\
\hline 3288,2 & 1,0 & K & 3205,6 & 0,8 & 3288,7 & 0,190 & 3 & 2,6 & 20 & 0,064 & 22 & 0,028 & 0,057 & 0,065 & M1(E2) & b \\
\hline 3352,8 & 1,5 & $\mathrm{~K}$ & 3270,5 & 0,9 & 3353,6 & 0,036 & 7 & 0,6 & 15 & 0,060 & 20 & 0,027 & 0,055 & 0,062 & M1 (E2) & $-L 3288$ \\
\hline & & $\mathrm{L}$ & 3337,6 & 1,4 & & 0,012 & 30 & & & 0,020 & 35 & & & & & $+K 3420 ?$ \\
\hline & & $\mathrm{K}$ & 3285,6 & 1,6 & 3368,7 & 0,012 & 30 & $<0,2$ & $-z$ & $>0,06$ & - & 0,026 & 0,054 & 0,061 & M1 & $\mathrm{w}$ \\
\hline $3459^{g}$ & $\mathrm{gr}$ & $\mathrm{K}$ & 3381,8 & 2,0 & 3464,9 & 0,006 & 35 & 0,15 & - & 0,04 & - & 0,025 & 0,052 & 0,057 & E $2, \mathrm{M} 1, \mathrm{E} 1$ & $\mathrm{w}$ \\
\hline 3500,2 & 1,0 & K & 3414,3 & 1,5 & 3497,4 & 0,009 & 45 & 0,47 & 20 & 0,020 & 60 & 0,025 & 0,051 & 0,056 & E1 & w \\
\hline $3578^{\mathrm{g}}$ & 5,0 & $\mathrm{~K}$ & 3502,5 & 2,4 & 3585,6 & 0,006 & 30 & $<0,2$ & -7 & $>0,03$ & - & 0,025 & 0,051 & 0,053 & E 1, E 2, M 1 & $w, p$ \\
\hline $3596^{\mathrm{g}}$ & 5,0 & $\mathrm{~K}$ & 3515,3 & 1,7 & 3598,4 & 0,01 & 20 & 0,23 & - & 0,04 & - & 0,024 & 0,049 & 0,052 & $\mathrm{E} 2, \mathrm{M} 1, \mathrm{E} 1$ & w \\
\hline 3633,4 & 1,0 & $\mathrm{~K}$ & 3548,0 & 1,8 & 3631,1 & 0,007 & 35 & 0,39 & 25 & 0,018 & 30 & 0,024 & 0,048 & 0,051 & E 1 & w \\
\hline $3654^{\mathrm{g}}$ & - & $\mathrm{K}$ & 3571,3 & 1,4 & 3654,4 & 0,009 & 20 & $\sim 0,2$ & - & 0,045 & - & 0,024 & 0,048 & 0,051 & $-\mathrm{E} 2, \mathrm{M} 1$ & b, $-L 3586$ \\
\hline $3713^{\mathrm{g}}$ & 6,0 & $\mathrm{~K}$ & 3635,6 & 2,1 & 3718,7 & 0,004 & 25 & $<0,2$ & -7 & $>0,02$ & - & 0,024 & 0,048 & 0,051 & $\mathrm{E} 1(\mathrm{E} 2, \mathrm{M} 1)$ & $-L 3654$ \\
\hline 3750,3 & 1,0 & $\mathrm{~K}$ & 3666,3 & 1,6 & 3749,4 & 0,007 & 35 & 0,31 & 30 & 0,022 & 40 & 0,023 & 0,046 & 0,048 & E 1 & $\mathrm{w}$ \\
\hline 3828,3 & 1,0 & $\mathrm{~K}$ & 3744,7 & 1,7 & 3827,8 & 0,016 & 10 & 0,32 & - & 0,048 & - & 0,022 & 0,044 & 0,045 & $\mathrm{M} 1, \mathrm{E} 2, \mathrm{E} 1$ & \\
\hline 3841,0 & 1,0 & K & 3759,8 & 1,6 & 3842,9 & 0,015 & 25 & 0,23 & - & 0,063 & - & 0,022 & 0,044 & 0,045 & $\mathrm{M} 1, \mathrm{E} 2$ & w \\
\hline 3869,1 & 1,5 & $\mathrm{~K}$ & 3784,8 & 1,3 & 3867,9 & 0,010 & 25 & 0,47 & 18 & 0,021 & 35 & 0,022 & 0,043 & 0,044 & E 1 & \\
\hline & & $\mathrm{K}$ & 3819,5 & 1,7 & 3902,7 & 0,005 & 30 & $<0,2$ & - & $>0,025$ & - & 0,022 & 0,042 & 0,043 & $\mathrm{E} 1, \mathrm{E} 2, \mathrm{M} 1$ & w, $-L 3827$ \\
\hline 3952,3 & 1,5 & $\mathrm{~K}$ & 3864,4 & 1,8 & 3947,5 & 0,007 & 30 & 0,40 & 25 & 0,017 & 40 & 0,021 & 0,041 & 0,041 & E 1 & \\
\hline 4018,4 & 1,5 & $\mathrm{~K}$ & 3933,8 & 1,4 & 4016,9 & 0,007 & 15 & 0,22 & 30 & 0,037 & 40 & 0,020 & 0,040 & 0,040 & $\mathrm{M} 1, \mathrm{E} 2, \mathrm{E} 1$ & $-L 3947$ \\
\hline 4072 & - & K & 3986,8 & 2,1 & 4069,9 & 0,005 & 45 & 0,17 & - & 0,030 & - & 0,020 & 0,039 & 0,039 & M1,E 2, E 1 & \\
\hline $4095^{\mathrm{c}}$ & & $\mathrm{K}$ & 4012,0 & 4,0 & 4095,1 & 0,004 & 30 & 0,26 & - & 0,015 & - & 0,020 & 0,039 & 0,038 & E 1 & \\
\hline 4119,3 & 1,0 & $\mathrm{~K}$ & 4032,3 & 1,4 & 4115,4 & 0,008 & 20 & 0,5 & 15 & 0,015 & 30 & 0,019 & 0,038 & 0,037 & E 1 & \\
\hline $4143^{\mathrm{g}}$ & 6,0 & $\mathrm{~K}$ & 4056,0 & 1,9 & 4139,1 & 0,007 & 25 & $<0,25$ & -1 & $>0,03$ & - & 0,019 & 0,038 & 0,037 & $\mathrm{M} 1, \mathrm{E} 2, \mathrm{E} 1$ & $\mathrm{w},+L 4070$ \\
\hline 4176,1 & 2,0 & $\mathrm{~K}$ & 4095,6 & 1,4 & 4178,7 & 0,006 & 30 & 0,28 & 20 & 0,020 & 40 & 0,019 & 0,038 & 0,036 & E1 1 & $-L 4115$ \\
\hline 4245,6 & 2,0 & $\mathrm{~K}$ & 4163,8 & 2,0 & 4246,9 & 0,004 & 30 & 0,24 & 20 & 0,017 & 40 & 0,019 & 0,036 & 0,034 & E 1 & w, $-L 4178$ \\
\hline 4273,2 & 1,0 & $\mathrm{~K}$ & 4189,2 & 1,4 & 4272,3 & 0,013 & 12 & 0,41 & 15 & 0,032 & 25 & 0,019 & 0,036 & 0,034 & M 1, E 2, E 1 & \\
\hline $4326^{\mathrm{g}}$ & 8,0 & $\mathrm{~K}$ & 4241,2 & 1,5 & 4324,3 & 0,007 & 15 & & & & & & & & & \\
\hline $4346^{\mathrm{g}}$ & 7,0 & K & 4264,0 & 1,7 & 4347,1 & 0,007 & 15 & & & & & & & & & \\
\hline 4373 & - & $\mathrm{K}$ & 4289,6 & 1,5 & 4372,7 & 0,016 & 8 & 0,76 & 15 & 0,021 & 20 & 0,018 & 0,035 & 0,033 & E1 & b \\
\hline $4381^{\mathrm{g}}$ & 7,0 & $\mathrm{~K}$ & 4304,3 & 2,1 & 4387,4 & 0,006 & 60 & & & & & & & & & $\mathrm{w}, \mathrm{b},+L 4324$ \\
\hline & & $\mathrm{K}$ & 4317,7 & 2,7 & 4400,8 & 0,003 & 40 & & & & & & & & & w \\
\hline & & K & 4338,1 & 2,4 & 4421,2 & 0,004 & 30 & & & & & & & & & w \\
\hline & & $\mathrm{K}$ & 4358,7 & 2,6 & 4441,8 & 0,002 & 35 & & & & & & & & & b, $-L 4372$ \\
\hline 4458,8 & 1,5 & $\mathrm{~K}$ & 4373,8 & 1,9 & 4456,9 & 0,005 & 15 & 0,31 & 15 & 0,017 & 25 & 0,017 & 0,034 & 0,031 & E 1 & $\mathrm{~b},-L 4387$ \\
\hline 4537,8 & 1,5 & K & 4451,5 & 1,6 & 4534,6 & 0,005 & 15 & 0,31 & 15 & 0,018 & 25 & 0,017 & 0,033 & 0,030 & E 1 & b \\
\hline
\end{tabular}




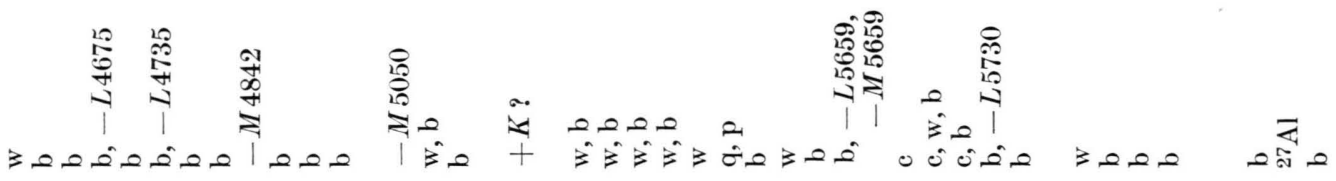

\begin{tabular}{|c|c|c|c|c|c|c|c|c|}
\hline 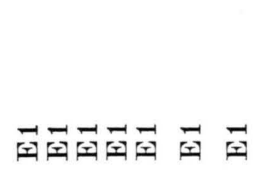 & 国可国 & 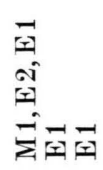 & $\begin{array}{l}\vec{z} \\
\text { â } \\
\text { â } \\
\text { â }\end{array}$ & 式 & $\overrightarrow{\mid c}$ & 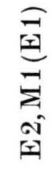 & 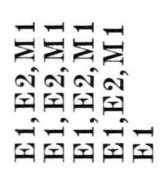 & 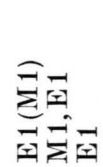 \\
\hline 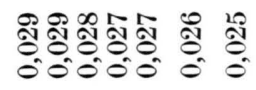 & 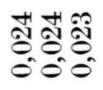 & 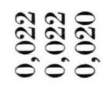 & จิ & $\stackrel{\infty}{0}$ & $\overrightarrow{0}$ & 등 & 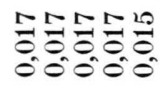 & 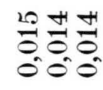 \\
\hline
\end{tabular}

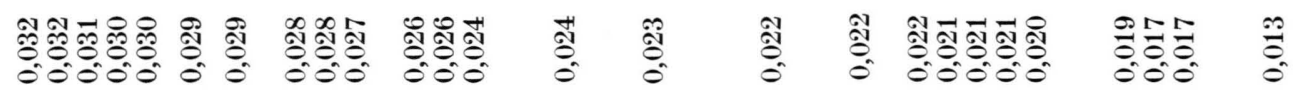

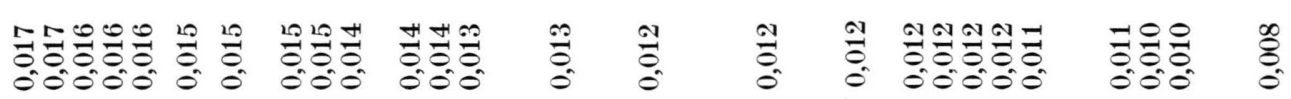

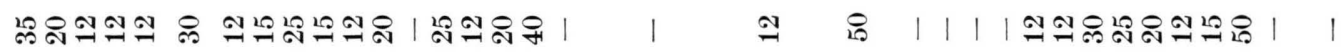

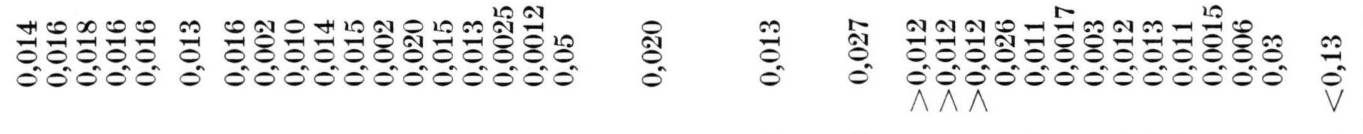

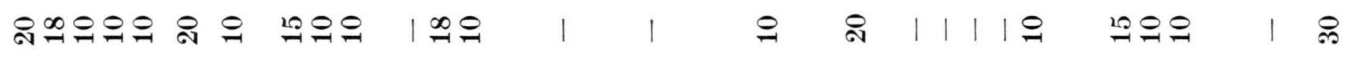

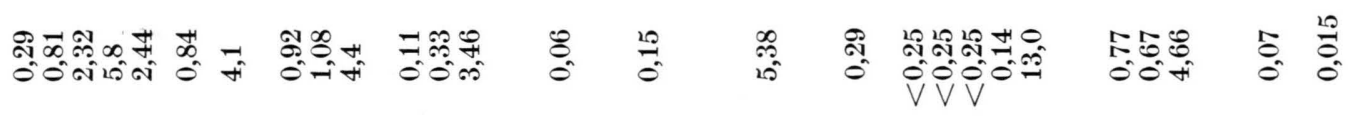

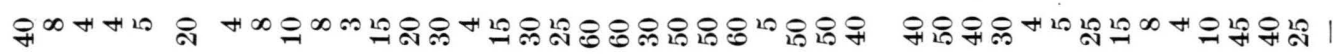

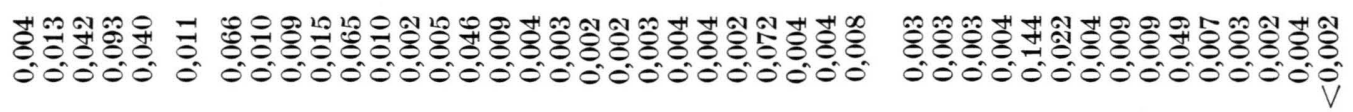

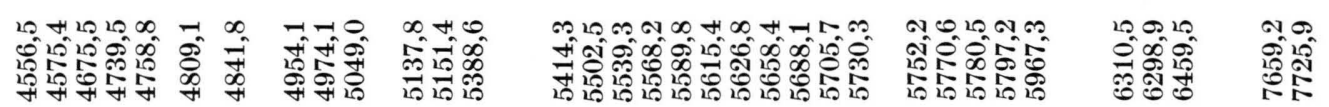

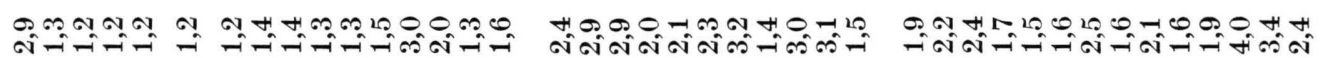

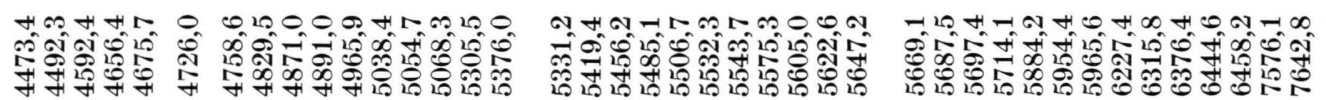

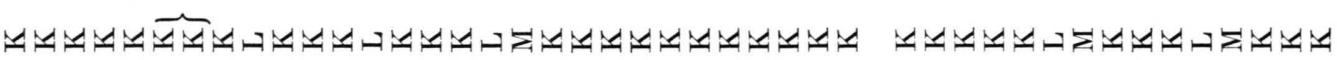

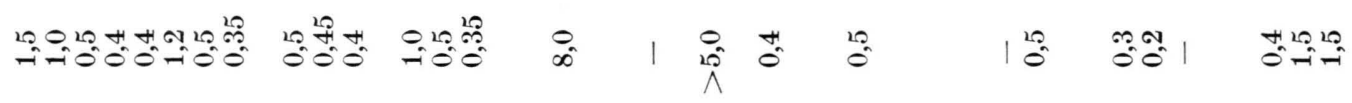

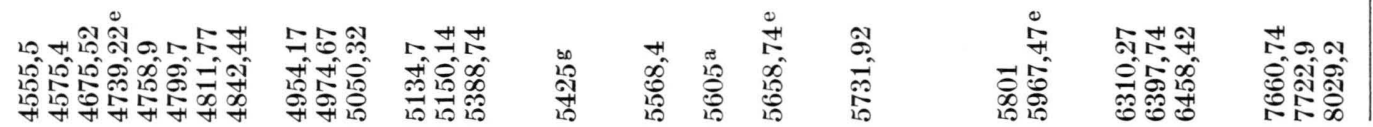


SCHult u. a. ${ }^{28}$ entnommen, falls Spalte 1 keine andere Bemerkung enthält, da diese Intensitäten sehr sorgfältig geeicht worden waren. Oberhalb $3,3 \mathrm{MeV}$ ist für die $\gamma$-Intensitäten in Spalte $9 \mathrm{der}$ Mittelwert aus den Messungen von Schult u. a. ${ }^{28}$, Groshev u. a. ${ }^{26}$ und RASMUSSEN u. a. ${ }^{32}$ angegeben. In Abb. 2 ist das Verhältnis der theoretischen Konversionskoeffizienten von $\mathrm{CHURCH}^{9} \mathrm{zu}$ den experimentell bestimmten Koeffizienten aufgetragen, wobei die verschiedenen $\gamma$-Messungen verwendet wurden. Diese $\gamma$-Intensitäten weichen auf Grund ihres Absoluteichfehlers von ca. 30\% untereinander zum Teil um den Faktor 2 ab. Bei der Mittelwertbildung wird der systematische Fehler wahrscheinlich kleiner werden. Trotzdem kann auch der Mittelwert der Intensitäten und damit der in Spalte 12 der Tabelle angegebene experimentelle Konversionskoeffizient noch einen systematischen Fehler von etwa $30 \%$ enthalten. Unter der Voraussetzung, daß die theoretischen Konversionskoeffizienten von Church richtig sind, scheint jedoch der systematische Fehler der experimentellen Konversionskoeffizienten (Spalte 12) nach Abb. 2 kleiner als 5\% zu sein. Die theoretischen Konversionskoeffizienten in Spalte 13, 14, 15 stammen bis $3000 \mathrm{keV}$ aus der Arbeit von SLIV und BAND ${ }^{14}$, bzw. sind durch lineare Extrapolation hieraus entstanden. Oberhalb $3000 \mathrm{keV}$ sind sie der
Arbeit von $\mathrm{CHURCH}^{9}$ entnommen. Alle $L$ - und $M$ Schalen-Koeffizienten stammen aus Ref. ${ }^{14}$, bzw. sind daraus linear extrapolierte Werte. In Spalte 16 bedeutet der Ausdruck M1 $+\mathrm{K} \% \mathrm{E} 2$ :

$$
(100-K) \% I(\mathrm{M} 1)+K \% I(\mathrm{E} 2) .
$$

\section{Hochenergetische Konversionskoeffizienten der K-Schale}

Abb. 3 enthält die experimentellen Konversionskoeffizienten $\alpha_{\mathrm{K}}$ von ${ }^{200} \mathrm{Hg}$ und die Tabellenwerte von $\mathrm{CHURCH}^{9}$ und Dragoun ${ }^{10}$. Die theoretischen Werte unterhalb von $2,5 \mathrm{MeV}$ sind der Arbeit von Sliv und BAND ${ }^{14}$ entnommen. Bei Energien von $7 \mathrm{MeV}$ liegen die berechneten $\alpha_{\mathrm{K}}$ über dem im doppelt logarithmischen Maßstab linear extrapolierten Werten von Sliv und Band. In Ref. 9 und 11 werden relativistische Wellenfunktionen für das freie und gebundene Elektron verwendet und Korrekturen für statische und dynamische endliche Kernausdehnungseffekte, sowie für die Abschirmungseffekte eingeführt.

Die Konversionskoeffizienten für hochenergetische M1- und El-Übergänge, die man nach dem thermischen Neutroneneinfang vom Einfangsniveau aus im wesentlichen erwartet, unterscheiden sich im Fall von ${ }^{200} \mathrm{Hg}$ bei $7 \mathrm{MeV}$ noch um den Faktor 1,75. Bei

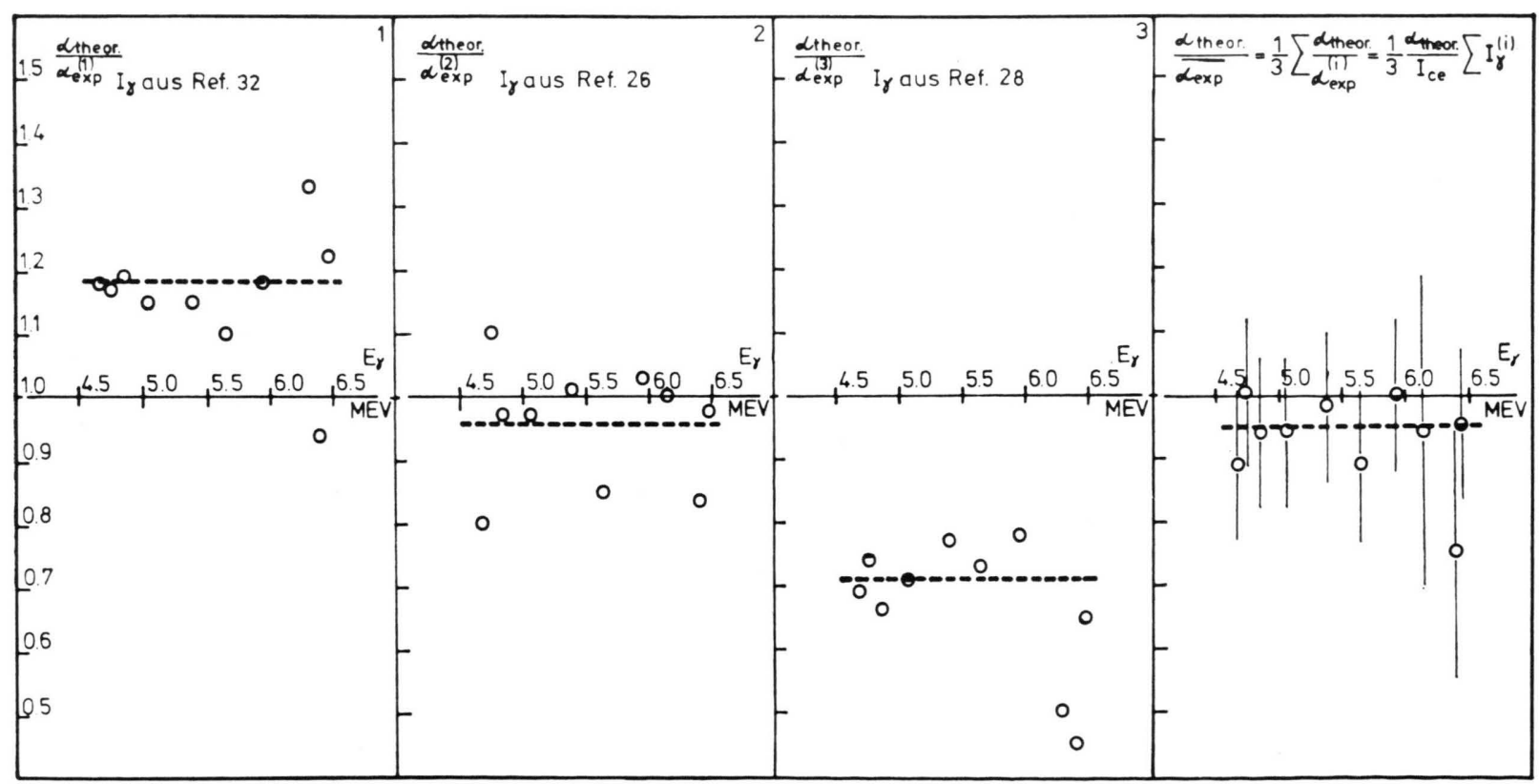

Abb. 2. Verhältnis der theoretischen zu den experimentellen K-Konversionskoeffizienten mit verschiedenen Gamma-Intensitäten. 


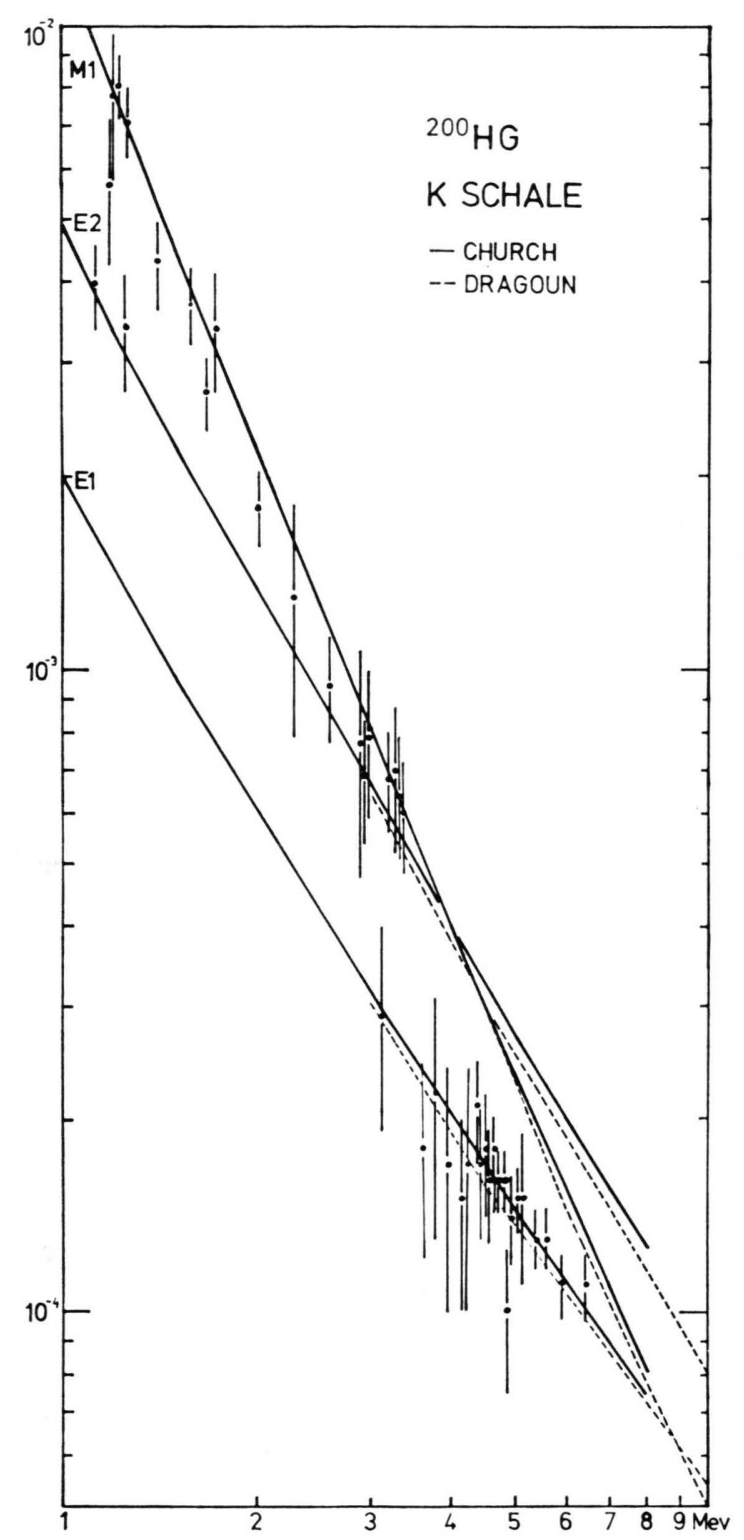

Abb. 3. Experimentelle und theoretische Konversionskoeffizienten der K-Schale von ${ }^{200} \mathrm{Hg}$.

den vorliegenden Fehlergrenzen lassen sich die gemessenen hochenergetischen Konversionskonffizienten eindeutig der Multipolordnung El oder Ml (E2) zuordnen. Über $3,5 \mathrm{MeV}$ gehören alle intensiveren Linien zu E1-Übergängen, unter $3 \mathrm{MeV}$ zu E2- und M1-Übergängen. Die gemessenen Konversionskoeffizienten bestätigen den Verlauf der theoretischen Werte. Der Schwerpunkt der Meßwerte liegt 39 O. W. B. Schult, private Mitteilung (1970). bei einer quadratischen Fehlerwichtung nur sehr wenig über der Kurve von Church. Wegen der bereits diskutierten möglichen Absolutverschiebung der Gamma- und Elektronenintensitäten und damit der Konversionskoeffizienten läßt sich nichts über ihre genauere Zuordnung zu einer der beiden Berechnungen aussagen. Wie man am Beispiel des ${ }^{114} \mathrm{Cd}$ sieht, unterscheiden sich auch die theoretischen Werte von Trusov ${ }^{11}$ und Carroll und O'Connell ${ }^{13}$ sehr wenig von $\mathrm{CHURCH}^{8}$ und Dragoun ${ }^{10}$. Als Korrekturgrößen bei El-Übergängen im ${ }^{200} \mathrm{Hg}$ gibt Church für die Abschirmung 2,3\% und für den statischen Effekt der endlichen Kernausdehnung kleiner als 5\% an. Für die Überprüfung dieser Größen sind die systematischen Meßfehler zu groß. Günstiger hierfür wären hochenergetische M1-Ưbergänge, für die die Korrekturgrößen ca. 30\% betragen. Auch die Durchdringungseffekte (penetration) gehen stärker in die M1- und E2-Konversionskoeffizienten ein als in die El-Koeffizienten. Nach $\mathrm{CHURCH}^{9}$ gilt für die Korrektur von $\alpha$ auf den Penetrationeffekt:

$$
\alpha=\alpha_{0} \cdot\left(1+\sum_{i} A_{i} \lambda_{i}+\ldots\right),
$$

wobei die Koeffizienten $A_{i}$ für ${ }^{200} \mathrm{Hg}<10 \%$ sind. Man erwartet also nur für Kernparameter $\lambda_{i}$, die über 1 liegen, eine meßbare Beeinflussung des Konversionskoeffizienten. Die gemessenen $\alpha_{K}$ weichen innerhalb ihres Fehlers nicht von den unkorrigierten Werten $\alpha_{0} \mathrm{ab}$, so daß in ${ }^{200} \mathrm{Hg}$ für alle intensiven Übergänge über $3,5 \mathrm{MeV}$ gilt:

$$
\sum_{i} A_{i} \lambda_{i}<0,15 \text {. }
$$

Die Konversionskoeffizienten der schwachen, in Abb. 3 nicht eingezeichneten Utbergänge liegen zwar über den Tabellenwerten $\alpha_{K}(E 1)$, ihre großen Fehler lassen aber keine Schlüsse über eine eventuelle Verschiebung durch Penetrationseffekte zu.

\section{Hochenergetische Konversionskoeffizienten der L- und M-Schale}

Das Konversionskoeffizientenverhältnis $\alpha_{K} / \alpha_{L}$ und damit $\alpha_{L}$ reagieren empfindlich auf die Korrekturgrößen der Abschirmung und der endlichen Kernausdehnung im theoretischen Konversionskoeffizientenansatz ${ }^{40}$. Allerdings wird diese Abhängigkeit mit zunehmendem $Z$ geringer werden, da die Abschir-

40 V. F. Trusov, Izv. Akad. Nauk SSSR, Ser. Fiz. 34, 1661 [1970]. 
mung der Hüllenelektronen relativ zur Kernladungszahl kleiner wird. Deswegen eignet sich ${ }^{200} \mathrm{Hg}$ nicht so gut zur Überprüfung dieser Korrekturgrößen wie ein leichterer Kern. Zudem sind die schwachen hochenergetischen $L$-Linien mit großen relativen Intensitätsfehlern behaftet.

Seit neuestem liegen neben den $L$-Schalen Konversionskoeffizienten von SLIV und BAND ${ }^{14}$, die für $E>2,5 \mathrm{MeV}$ extrapoliert werden müssen, Berechnungen von TRUSOV ${ }^{40}$ und Dragoun ${ }^{10}$ für Energien bis 10 bzw. $20 \mathrm{mc}^{2}$ vor. Diese neuen Werte für die $L_{1}$-Schale werden in Abb. 4 mit den gemessenen Konversionskoeffizienten verglichen. Von den $L$-Linienintensitäten wurde der $L_{2}$ - und $L_{3}$-Beitrag abgezogen. Dragoun gibt diesen Beitrag für $Z=80$ und El-Übergänge bei $5 \mathrm{MeV}$ mit

$$
\left(L_{2}+L_{3}\right) / L_{1}=0,07
$$

an. Von $L$-Linien, die nicht von den zugehörigen $M$-Linien getrennt waren, wurde auch der $M$-Beitrag nach Dragoun subtrahiert (für $Z=80$ und E1-Übergänge bei $5 \mathrm{MeV}: L / M=5$ ). In Abb. 4 sieht man, daß für die stärksten Übergänge eine eindeutige Zuordnung zu den Multipolordnungen Ml oder El möglich ist. Die Abweichungen der Tabellenwerte untereinander sind sehr klein gegen die Fehler der gemessenen Koeffizienten.

Nach den Berechnungen von Dragoun ist das Verhältnis $\alpha_{\mathrm{K}} / \alpha_{\mathrm{L}}$ über $3 \mathrm{MeV}$ für die einzelnen Multipolordnungen annähernd energieunabhängig. Für $Z=80$ erhält man durch lineare Interpolation und nach einer einfachen Umrechnung aus Ref. ${ }^{10}$ die in Tab. 2 aufgeführten Werte für $\alpha_{\mathrm{K}} / \alpha_{\mathrm{L}}$.

Tab. 2. Das Verhältnis $\alpha_{\mathrm{K}} / \alpha_{\mathrm{L}}$ nach DragouN ${ }^{10}$ für $Z=80$.

\begin{tabular}{cccc}
\hline$E[\mathrm{MeV}]$ & E 1 & E 2 & M 1 \\
\hline 3 & 6,9 & 6,5 & 6,5 \\
10 & 7,0 & 6,9 & 6,6 \\
\hline
\end{tabular}

Tab. 3. Experimentelle Werte des Verhältnisses $\alpha_{K} / \alpha_{L}$.

\begin{tabular}{cccc}
\hline$E \gamma[\mathrm{keV}]$ & 5389 & 5967 & 6458 \\
\hline$\alpha_{K} / \alpha_{L}$ & $5.4 \pm 15 \%$ & $6.6 \pm 6 \%$ & $7 \pm 11 \%$ \\
\hline
\end{tabular}

Aus den gemessenen hochenergetischen Verhältnissen $\alpha_{K} / \alpha_{L}$ für El-Übergänge (Tab. 3) erhalten wir als Mittelwert mit quadratischer Fehlerwichtung:

$$
\alpha_{K} / \alpha_{L}(\mathrm{E} 1)=6,5 \pm 5 \% \text {. }
$$

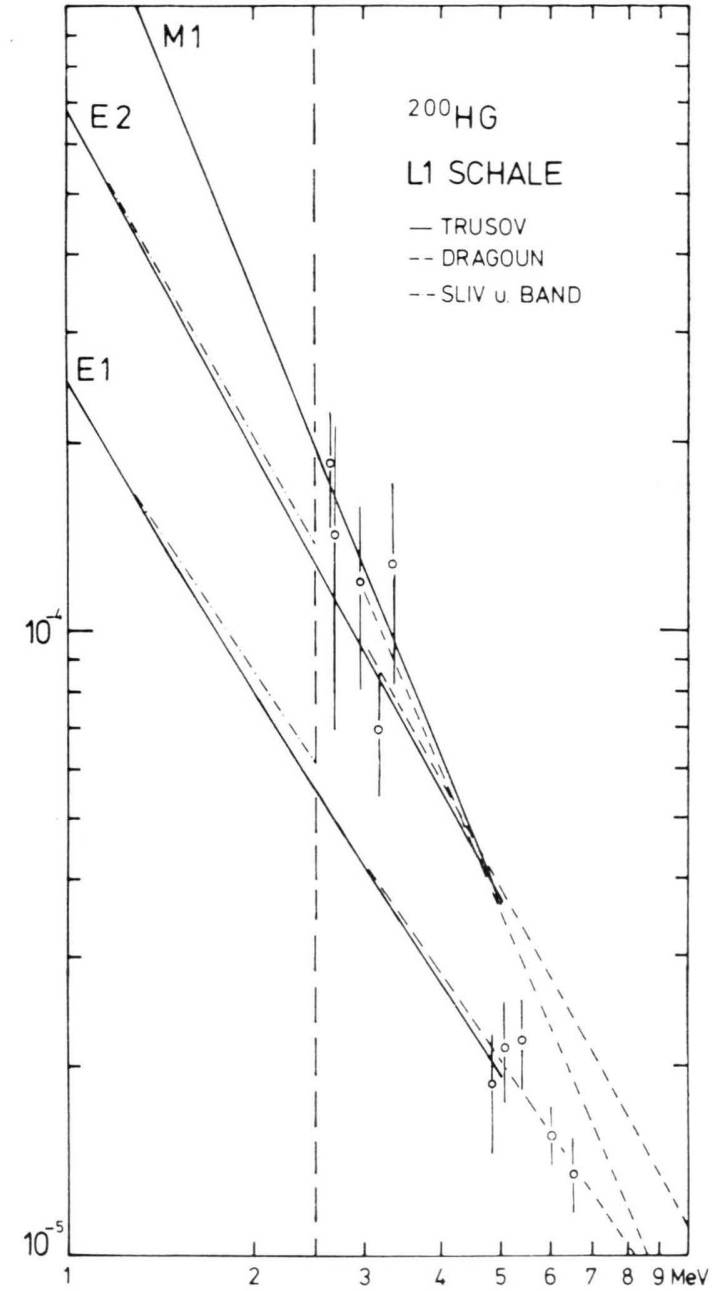

Abb. 4. Experimentelle und theoretische Konversionskoeffizienten der $\mathrm{L}_{1}$-Schale von ${ }^{200} \mathrm{Hg}$.

Dieser Wert stimmt mit dem von Dragoun berechneten gut überein. Eine genauere Überprüfung der Ansätze und Näherungen für die Korrekturgrößen in der theoretischen Berechnung der Koeffizienten ist nicht möglich. Hierfür wären Ml-Übergänge empfindlicher.

In der Messung sind $L$ - und $M$-Linien nicht als isoliert stehende Linien aufgelöst. Sie werden erst durch den Computer-Fit getrennt. Die Intensitätsfehler für die schwachen $M$-Linien sind deshalb beträchtlich. Aus diesem Grund wird nicht das $L / M$-Verhältnis direkt, sondern nur das von DRAGOUN $^{\mathbf{1 0}}$ für $Z=80$ linear interpolierte Verhältnis $K /(L+M)$ diskutiert (Tab. 4). Dieses Verhältnis läßt sich mit sehr kleinem Fehler angeben und ermöglicht daher eine weitere experimentelle Überprü- 
fung für den theoretischen Ansatz des Abschirmeffektes.

Tab. 4. Verhältnis $\alpha_{K} /\left(\alpha_{L}+\alpha_{M}\right)$ nach DRAGouN ${ }^{10}$ für $Z=80$.

\begin{tabular}{cccc}
\hline$E[\mathrm{MeV}]$ & $\mathrm{E} 1$ & $\mathrm{E} 2$ & $\mathrm{M} 1$ \\
\hline 3 & 5,6 & 5,1 & 5,3 \\
10 & 5,8 & 5,5 & 5,4 \\
\hline
\end{tabular}

Die experimentellen $\alpha_{K} /\left(\alpha_{L}+\alpha_{M}\right)$-Werte stehen in Tab. 5.

Als Mittelwert ergibt sich

$$
\alpha_{K} /\left(\alpha_{L}+\alpha_{M}\right)(\mathrm{E} 1)=5,5 \pm 5 \% .
$$

Dieser Wert besagt, daß der Ansatz für die Korrekturen der Abschirmung und der endlichen Kernausdehnung von Dragoun durch das Experiment für hohe Energien gut bestätigt wird.

Für das experimentelle Verhältnis $\alpha_{L} / \alpha_{M}$ bei $5 \mathrm{MeV}$ erhält man somit für $Z=80$ aus den Meßwerten:

$$
\frac{K /(L+M)}{K / L}=\frac{6,5 \pm 5 \%}{5,5 \pm 5 \%}
$$

Daraus folgt:

$$
L / M=5,5 \pm 10 \% .
$$

Aus den Tabellen von Dragoun läßt sich errechnen:

$$
L / M=4,9 \text {. }
$$

\section{Niveauschema}

Über das Niveauschema von ${ }^{200} \mathrm{Hg}$ existieren viele umfangreiche Arbeiten aus dem ${ }^{200} \mathrm{Tl}{ }^{23},{ }^{24}$ und ${ }^{200} \mathrm{Au}{ }^{19,}{ }^{21}$ Zerfall und nach dem Einfang thermischer Neutronen 28, 29, 33, 34. In der vorliegenden Arbeit wird im wesentlichen das Schema von Schult u. a. ${ }^{28}$ bestätigt und ergänzt. Beim Aufbau des Niveauschemas werden außer den eigenen Messungen die $\gamma$-Linien von MAIER u. a. ${ }^{27}$ und Schult u. a. ${ }^{28}$ verwendet. Das Niveauschema wurde mit Hilfe folgender Argumente für die Energie, den Spin und die Parität der Niveaus aufgebaut: Energiekombi- nation der Übergänge, Multipolordnung der Übergänge und der Vergleich der Verzweigungsverhältnisse nach dem ${ }^{200} \mathrm{Tl}$-Zerfall und Neutroneneinfang. Es wurde außerdem darauf geachtet, daß alle Energien, Spins und Paritäten in Übereinstimmung mit anderen Meßergebnissen sind. Im Vergleich zum Niveauschema von Schult u. a. wurden viele neue Linien eingeordnet, zu zahlreichen Niveaus Spin und Parität bestimmt und 14 neue Niveaus hinzugefügt. Das gesamte Niveauschema enthält 47 Zustände und 145 Übergänge. Damit ist der größte Teil aller bekannten Linien in das Schema eingeordnet.

Eine Wahrscheinlichkeitsbetrachtung zeigt, daß z. B. zwischen die 12 Niveaus im Energiebereich von 1550 bis $1900 \mathrm{keV}$ jede der $14 \gamma$-Linien mit einer Sicherheit von $97 \%$ richtig eingeordnet ist. Von den 53 Linien über $1 \mathrm{MeV}$, die die Niveaus zwischen 2 und $4 \mathrm{MeV}$ entvölkern, können im Mittel 3 durch zufällige Energiekombination eingeordnet worden sein. Die Kenntnis der Multipolordnungen setzt die Unsicherheit der Einordnung herab. Um jedoch über $2 \mathrm{MeV}$ keine Niveaus einzuführen, die nur auf zufälliger Kombination beruhen, wurden nur solche in das Schema aufgenommen, die durch einen Übergang vom Einfangzustand bevölkert werden. Dabei wurde davon ausgegangen, daß alle Linien mit einer Energie von mehr als $4,4 \mathrm{MeV}$ vom Einfangzustand ausgehen.

In Tab. 6 sind alle Niveaus sowie die Besetzung von höheren Niveaus und die Entvölkerung zu niederen Niveaus aufgeführt. Es ist eine Korrelation zwischen dem Spin und der Bevölkerung der Niveaus festzustellen, die statistischen Berechnungen ${ }^{42}$ einigermaßen entspricht. Aus diesem Grunde kann die Besetzung eines Niveaus als Argument für die Spinzuordnung verwendet werden. Auf Grund dieser Betrachtung hat von den Niveaus bei $1845,96 \mathrm{keV}$ und $1882,98 \mathrm{keV}$, für die beide der Spin 2 oder 3 in Frage kommt, das erste mit gröBerer Wahrscheinlichkeit den Spin 3 und das zweite den Spin 2.

Tab. 5. Experimentelle Werte des Verhältnisses $\alpha_{K} /\left(\alpha_{L}+\alpha_{M}\right)$.

\begin{tabular}{clllll}
\hline$E(\mathrm{keV})$ & 4842 & 5050 & 5389 & 5967 & 6458 \\
\hline$\alpha_{K} /\left(\alpha_{L}+\alpha_{M}\right)$ & $7,2 \pm 15 \%$ & $6,6 \pm 15 \%$ & $5,1 \pm 15 \%$ & $5,5 \pm 7 \%$ & $4,9 \pm 11 \%$ \\
\hline
\end{tabular}

41 L. V. Groshev, A. M. Demidov, V. I. Sokolovskit, B. A. Bartholomew, A. Doveika, K. M. Eastwood u. S. MoNARO, Nuclear Data A 5, 243 [1969].
42 T. v. EgIDY, in: Neutron Capture Gamma-Ray Spectroscopy, IAEA Wien 1969, S. 541. 
Tab. 6. Liste der Niveaus und deren Besetzung.

\begin{tabular}{|c|c|c|c|}
\hline $\begin{array}{c}\text { Energie und } \\
\text { Fehler } \\
{[\mathrm{keV}]}\end{array}$ & $\begin{array}{c}\text { Spin } \\
\text { und } \\
\text { Parität }\end{array}$ & $\begin{array}{l}\text { Summe der } \\
\text { Intensitäten } \\
\text { zu niederen } \\
\text { Niveaus } \\
\text { pro } 100 \text { Einf. }\end{array}$ & $\begin{array}{c}\text { Besetzung } \\
\text { von bekann- } \\
\text { ten höheren } \\
\text { Niveaus } \\
\text { pro } 100 \text { Einf. }\end{array}$ \\
\hline 0 & $0^{+}$ & - & 98,0 \\
\hline $367,97 \pm 0,02$ & $2^{+}$ & 74,0 & 57,0 \\
\hline $947,39 \pm 0,06$ & $4^{+}$ & 2,1 & 0,8 \\
\hline $1029,43 \neq 0,06$ & $0^{+}$ & 6,0 & 2,8 \\
\hline $1254,26 \pm 0,06$ & $2^{+}$ & 5,5 & 1,5 \\
\hline $1570,38 \mp 0,06$ & $1^{+}$ & 11,2 & 7,1 \\
\hline $1273,75 \pm 0,06$ & $2^{+}$ & 3,5 & 0,6 \\
\hline $1593,45 \pm 0,10$ & $1^{+}, 2^{+}$ & 2,9 & 0,5 \\
\hline $1631,03+0,08$ & $1^{+}$ & 5,1 & 1,2 \\
\hline $1641,50 \pm 0,08$ & $2^{+}$ & 3,0 & 0,7 \\
\hline$(1713,0 \pm 0,8)$ & $\left(4^{-}, 5^{-}\right)$ & 0,3 & - \\
\hline $1718,38 \pm 0,08$ & $1^{+}$ & 2,9 & 0,9 \\
\hline $1730,95 \pm 0,08$ & $2^{+}$ & 2,3 & 2,4 \\
\hline $1775,69 \pm 0,08$ & $3^{+}$ & 0,5 & - \\
\hline $1845,92 \pm 0,08$ & $\left(2^{+}\right) 3^{+}$ & 0,3 & 0,1 \\
\hline $1856,97+0,08$ & $0^{+}$ & 1,1 & \\
\hline $1882,98 \pm 0,08$ & $2^{+}\left(3^{+}\right)$ & 1,2 & \\
\hline $2061,40 \pm 0,08$ & $1^{+}$ & 15,0 & 14,0 \\
\hline $2228,9 \pm 0,10$ & $\left(1^{+}\right)$ & 0,2 & 0,1 \\
\hline $2248,8 \pm 0,3$ & $1^{+}$ & 0,6 & \\
\hline $2259,1 \pm 0,5$ & & & \\
\hline $2297,3 \pm 0,7$ & $\left(1^{+}\right)$ & 0,9 & 0,3 \\
\hline $\begin{array}{l}(2324,4 \pm 2) \\
2370,40 \pm 0,25\end{array}$ & $1+$ & & \\
\hline$(2401 \quad \pm 1)$ & 1 & & \\
\hline$(2439,6 \pm 1,5)$ & & & \\
\hline$(2459,7 \pm 1,5)$ & $\left(1^{+}\right)$ & 0,7 & \\
\hline 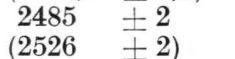 & $\left(1^{+}\right)$ & & \\
\hline $2639,7 \pm 0,3$ & $1^{+}$ & 4,9 & 3,5 \\
\hline$(2878,2 \pm 0,25)$ & $1^{+}$ & 2,1 & 0,33 \\
\hline $2894,4 \pm 0,5$ & $1^{+}$ & 0,14 & 0,11 \\
\hline $2978,33 \pm 0,2$ & $1^{+}$ & 2,6 & 4,4 \\
\hline $3053,5 \pm 0,5$ & $1^{+}$ & 1,6 & 1,1 \\
\hline $3074,7 \pm 0,4$ & $1^{+}$ & 0,6 & 0,9 \\
\hline $3186,1 \pm 0,3$ & $1^{+}$ & 3,2 & 4,1 \\
\hline $3217,1 \pm 0,6$ & $1^{+}$ & 0,7 & 0,6 \\
\hline$(3230 \pm 1)$ & $1^{+}$ & & 0,11 \\
\hline $3269,5 \pm 0,4$ & $1^{+}$ & 1,8 & 2,44 \\
\hline $3289,1 \pm 0,2$ & $1^{+}$ & 4,3 & 5,8 \\
\hline $3353,2 \pm 0,7$ & $1^{+}$ & 1,0 & 2,3 \\
\hline $3452,5 \pm 1$ & $1^{+}$ & & 0,8 \\
\hline $3492,1 \pm 1,2$ & $1^{+}$ & & 0,31 \\
\hline$(3572)$ & $1^{+}$ & 0,3 & 0,31 \\
\hline (3586 & & & \\
\hline 3637 & & 0,7 & \\
\hline $3655,1 \pm 1,5$ & $1^{+}$ & & 0,76 \\
\hline $8028,7 \pm 0,5$ & $0^{-}\left(1^{-}\right)$ & 100,0 & \\
\hline
\end{tabular}

Da die meisten Niveaus im einzelnen in früheren Arbeiten diskutiert worden sind, soll das hier nicht wiederholt werden. Es werden nur zwei neue Niveaus von besonderem Interesse besprochen.

$$
\text { Das 1713,0 keV (4-, } \left.5^{-}\right) \text {-Niveau }
$$

Aus theoretischen Überlegungen ${ }^{36}$ und dem Vergleich mit benachbarten Kernen ${ }^{21}$ erwartet man, daß die niedrigsten Zustände mit negativer Parität die Spins 4, 5, 6 und 7 haben. Sie werden mit Hilfe der $h_{11 / 2}$-Protonen-Loch-Konfiguration gebildet. Ton und Mitarbeiter ${ }^{21}$ fanden kürzlich durch verzögerte Koinzidenzmessung beim Zerfall des 18,7 h Isomers von ${ }^{200} \mathrm{Au}$ einen $\left(4^{-}, 5^{-}\right)$-Zustand bei $1707 \mathrm{keV}$. Dieses Niveau zerfällt durch einen $760 \mathrm{keV}$-Übergang zum $947 \mathrm{keV}-4^{+}$-Zustand. Im $\gamma$-Spektrum von MAIER u. a. ${ }^{27}$ findet sich eine $765,6 \mathrm{keV}$-Linie, die im Konversionselektronenspektrum nicht beobachtet wird. Eine Abschätzung des Konversionskoeffizienten ergibt, daß es sich um einen El-Übergang handeln muß. Da dies der einzige bisher beobachtete niederenergetische E1-Übergang in ${ }^{200} \mathrm{Hg}$ ist und seine Intensität der erwarteten Bevölkerung eines $\left(4^{-}, 5^{-}\right)$-Niveaus entspricht, ordnen wir diese Linie als Übergang zwischen das $1713,0 \mathrm{keV}\left(4^{-}, 5^{-}\right)$-Niveau und das $947,36 \mathrm{keV} 4^{+}$-Niveau ein. Dieses Niveau kann jedoch nicht als gesichert gelten. Nach Covello und SATORIs ${ }^{36}$ liegt der $5^{-}$-Zustand unterhalb des $4^{-}$-Zustandes und hat die Energie

$$
E_{5}-=0,87+\Delta_{11 / 2} \text {. }
$$

Unter der Annahme, daß es sich bei dem $1713 \mathrm{keV}$ Niveau um diesen $5^{-}$-Zustand handelt, ergibt sich die Einteilchen-Protonen-Loch-Energie für den $h_{11 / 2^{-}}$ Zustand in ${ }^{200} \mathrm{Hg}$ zu $\Delta_{11 / 2}=\varepsilon_{11 / 2}-\varepsilon_{1 / 2}=840 \mathrm{keV}$. Bei den Nachbarkernen findet man ähnliche Werte ${ }^{36,21}$.

\section{Das $1856,97 \mathrm{keV}, 0^{+}$-Niveau}

Der $1857,1 \mathrm{keV}$ E0-Übergang wurde von GRosHEV u. a. ${ }^{26}$, SCHUlt u. a. ${ }^{33}$ und in der vorliegenden Messung gefunden. Da es sehr unwahrscheinlich ist, daß ein fast reiner E0-Übergang zu einem Niveau geht, das einen anderen Spin als 0 hat, muß man diese Linie entweder auf den Grundzustand oder auf das $1029 \mathrm{keV}$-Niveau setzen. Für ein $0^{+}$-Niveau bei $1856,97 \mathrm{keV}$ findet man vier Linien zu niederen Niveaus, deren Multipolordnung in Übereinstimmung mit dem $0^{+}$-Charakter ist. Die $1489,6 \mathrm{keV}$ Linie zum $367 \mathrm{keV}$-Niveau wurde zunächst nur im Konversionselektronenspektrum beobachtet. Im $\gamma$ Spektrum von Schult u. a. ${ }^{28}$ erscheint eine Linie bei der entsprechenden Energie, die jedoch von den Autoren offensichtlich als single escape peak des $2002 \mathrm{keV}$-Übergangs gedeutet wurde. Da das Verhältnis von single escape peak zu double escape peak energieunabhängig ist, kann man durch Vergleich der escape-Linien der $1693 \mathrm{keV}$ - und 2002-Übergänge den Anteil des single escape peaks am 


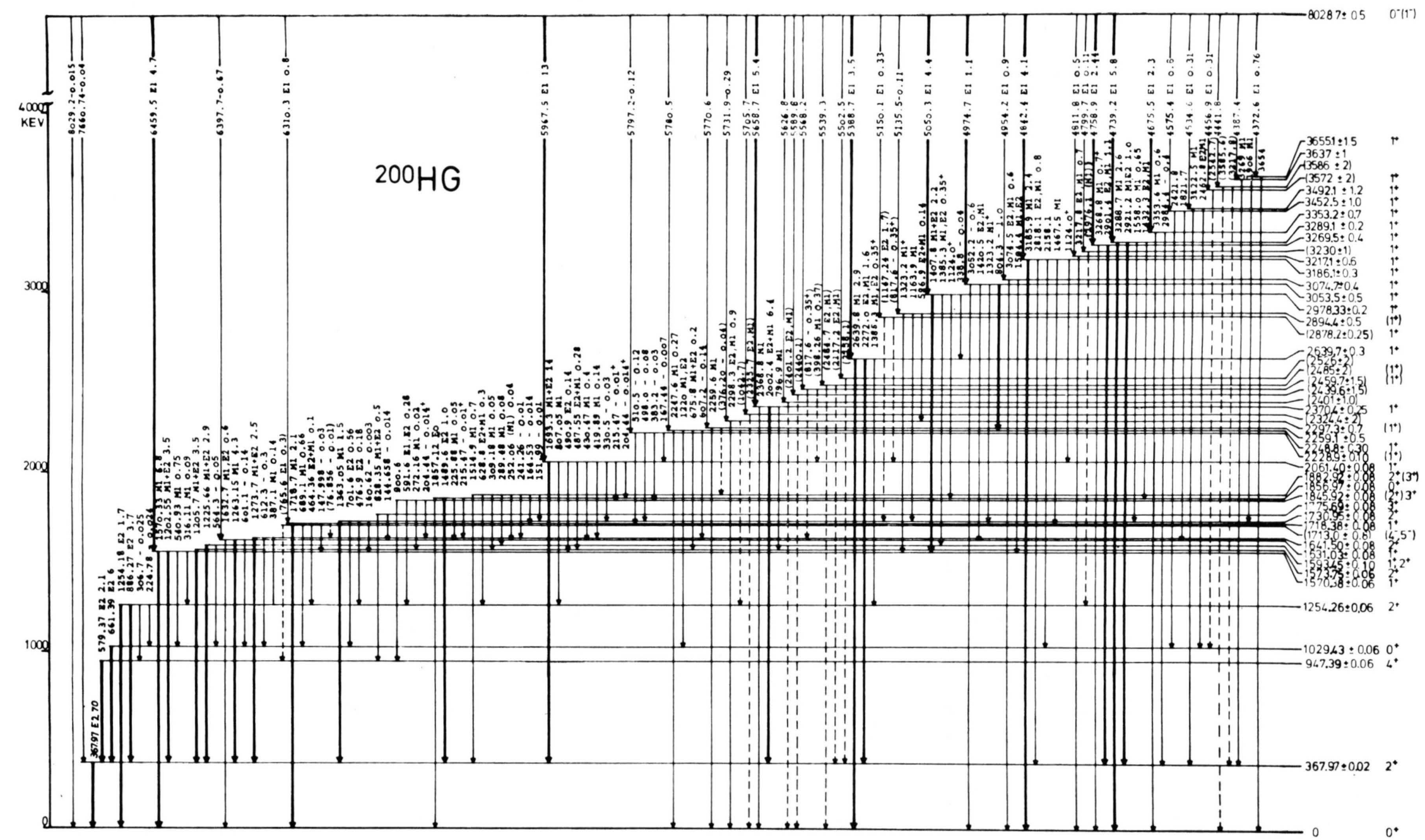

Abb. 5. Niveauschema von ${ }^{200} \mathrm{Hg}$ (Linien mit + sind zweimal eingeordnet, zu jeder Linie ist die Energie, die Multipolordnung und die Gamma-Intensität angegeben). 
1489,6 keV-Übergang abschätzen. Es ergibt sich eine Intensität für diesen Übergang von $1,0 \pm 0,3$ pro 100 Einfänge und damit ein Konversionskoeffizient, der die Multipolordnung E2 festlegt. Wegen dieser guten Übereinstimmung ist das 1856,97 keV-Niveau gesichert. Auch theoretische Berechnungen ${ }^{36}$ lassen das dritte $0^{+}$-Niveau bei etwa dieser Energie erwarten. Der starke E0-Übergang dieses 3-Phononenzustands zum Grundzustand ist zwar nach dem reinen Vibrationsmodell verboten, doch deutet schon die starke Aufspaltung des 2-Phononenzustands auf eine größere Störung hin.

Die Tatsache, daß man im Niveauschema von ${ }^{200} \mathrm{Hg}$ fast nur Zustände in positiver Parität findet, die meistens den Spin 1 oder 2 haben, läßt sich auf Grund der zur Verfügung stehenden EinteilchenZustände leicht verstehen. Da die Protonen-GapEnergie $\left(\Delta_{\mathrm{p}}=0,415 \mathrm{MeV}\right)$ bei ${ }^{200} \mathrm{Hg}$ geringer ist als

43 K. Kumar u. M. Baranger, Nucl. Phys. A 110, 529 [1967].

44 S. G. NiLsson, private Mitteilung (1970). die Neutronen-Gap-Energie $\quad\left(\Delta_{\mathrm{n}}=0,774 \mathrm{MeV}\right)^{43}$, erwartet man, daß die Protonenpaare zuerst aufbrechen. Das ergibt Niveaus, die aus den $s_{1 / 2^{-}}$und $d_{3 / 2}$-Protonen-Zuständen gebildet werden und deshalb positive Parität haben. Auch die Niveaus, die durch Aufbrechen von Neutronenpaaren entstehen, werden positive Parität und niedrigen Spin besitzen, weil sie sich aus $p_{1 / 2^{-}}, f_{5 / 2^{-}}$und $p_{3 / 2^{-}}$Zuständen zusammensetzen.

Prof. Dr. H. Daniel und Dr. H. Vonach danken wir herzlich für die stets gewährte Unterstützung. Dr. O. W. B. Schult sind wir für viele wesentliche Diskussionen und Anregungen zu großem Dank verpflichtet. Dr. E. CHurch sind wir für die Berechnung der Konversionskoeffizienten und Dr. G. HolzWARTH und Dr. M. Kleber für theoretische Hinweise dankbar. Die Betriebsgruppe des FRM-Reaktors ist unseren Wünschen immer entgegengekommen, wofür wir ihr danken.

45 G. A. Bartholomew u. a., Nuclear Data A 3, 385 [1967]. 